ACCEPTED MANUSCRIPT

\title{
Pattern formation in spin-coating of hybrid colloids in different magnetic field configurations
}

To cite this article before publication: Raheema Aslam et al 2019 J. Phys. D: Appl. Phys. in press https://doi.org/10.1088/1361-6463/ab2795

\section{Manuscript version: Accepted Manuscript}

Accepted Manuscript is "the version of the article accepted for publication including all changes made as a result of the peer review process, and which may also include the addition to the article by IOP Publishing of a header, an article ID, a cover sheet and/or an 'Accepted

Manuscript' watermark, but excluding any other editing, typesetting or other changes made by IOP Publishing and/or its licensors"

This Accepted Manuscript is @ 2019 IOP Publishing Ltd.

During the embargo period (the 12 month period from the publication of the Version of Record of this article), the Accepted Manuscript is fully protected by copyright and cannot be reused or reposted elsewhere.

As the Version of Record of this article is going to be / has been published on a subscription basis, this Accepted Manuscript is available for reuse under a CC BY-NC-ND 3.0 licence after the 12 month embargo period.

After the embargo period, everyone is permitted to use copy and redistribute this article for non-commercial purposes only, provided that they adhere to all the terms of the licence https://creativecommons.org/licences/by-nc-nd/3.0

Although reasonable endeavours have been taken to obtain all necessary permissions from third parties to include their copyrighted content within this article, their full citation and copyright line may not be present in this Accepted Manuscript version. Before using any content from this article, please refer to the Version of Record on IOPscience once published for full citation and copyright details, as permissions will likely be required. All third party content is fully copyright protected, unless specifically stated otherwise in the figure caption in the Version of Record.

View the article online for updates and enhancements. 


\title{
Pattern formation in spin-coating of hybrid colloids in different magnetic field configurations
}

\author{
R Aslam ${ }^{1} \ddagger$ \& W González-Viñas ${ }^{1,2}$
}

1 Universidad de Navarra, Complex System Group, Pamplona, Spain

${ }^{2}$ Universidad de Navarra, PHYSMED group, Pamplona, Spain

E-mail: wens@unav.es

5 May 2019

Abstract. We report experimental results on the patterns that are formed during spin-coating of magnetic colloids at moderate concentrations and compare them with results obtained in diluted colloids. We show that, for moderate concentrations, the magnetic interaction between the (ferro)magnetic particles and with the external field is strong enough to overcome the centrifugal force. We study two different configurations for the magnetic field. The first one consists on an axial uniform field, where we obtain spikes perpendicular to the substrate with a well defined order which decreases as rotation rate increases. The second one consists on a radial non-uniform field, where we obtain elongated deposits radially disposed on the substrate. The effect of magnetic fields at moderate concentrations on the effective viscosity is confirmed to be much more important in the case of a uniform magnetic field, by increasing the hydrodynamic time-scale which gives the ferromagnetic particles enough time to strongly interact to form the spikes.

Keywords: spin-coating, hybrid colloids, magnetoviscosity, colloidal structures

Submitted to: J. Phys. D: Appl. Phys.

\section{Introduction}

Spin-coating technique has been widely used to fabricate thin films on flat substrates [1-12]. This technique is simple, fast, very reproducible and requires a small amount of material. The formation of ordered colloidal crystals has been a challenge in a simple, fast and reproducible way. Spin-coating, if properly taylored, could be a suitable option to deposit colloidal films [1,13-18].

However, it has been shown that crystalline order in spin-coated colloids cannot be achieved easily due to the intrinsic symmetries of the process [19]. Alternative paths

† Present address: Electrical Engineering Division, Higher Colleges of Technology, United Arab Emirates. E-mail: raslam@hct.ac.ae 


\section{Pattern formation in magnetic spin-coating of hybrid colloids}

have been taken in order to modify the classical spin-coating, that is to say: by using patterned substrates [20], and by applying external fields during the process [21-23]. Both types of methods show that it is possible to break the axial symmetry that leads to Orientationally Correlated Polycrystals [19]. Nevertheless, up to this date, these techniques have not been able to substantially increase the average correlation length of the colloidal films.

At the same time, application of external fields during the spin-coating of colloids sensitive to those fields have emerged on the one hand as a new technique to measure rheological properties of colloids when the conditions are against the use of classical techniques (e.g. when the sedimentation time-scale is shorter than the measurement time) [23,24], and on the other hand to form specific height colloidal films by modifying the effective viscosity of the system. Besides, all these results allow to understand the spin-coating process in complex colloids (effective evaporation rate and its mechanisms, etc).

Pichumani et al. $[21,23]$ have studied the effect of homogeneous external magnetic fields on the spin coating of diluted superparamagnetic colloids. They proved that the sole effect of the magnetic field is to increase the effective viscosity, by using a continuum model [25] modified to incorporate the particulate nature of the colloid. Later on, Aslam et al. [24] showed that at low concentrations the results were valid for ferromagnetic particles. They have also investigated the behavior of moderately concentrated superparamagnetic colloids in different magnetic fields [20], where they found that the magnetoviscosity in inhomogeneous fields is strongly influenced by the magnetophoretic effect that increases the flows of magnetic fluids in the applied field direction.

For the sake of clarity, we summarize in the following the main assumptions and results of the preceding models. The original Cregan's model [25] assumes a Newtonian fluid system (solvent + solute) in the framework of the lubrication approximation, where evaporation rate does not depend on the rotation rate $\omega$. As a result, it gives the thickness of the deposited film $\mathrm{h}_{\infty}^{(s)}$ :

$$
h_{\infty}^{(s)}=\frac{C}{1-C}\left(\frac{3}{2} \nu E\right)^{\frac{1}{3}} \omega^{-\frac{2}{3}},
$$

where $C$ is the volume fraction, $\omega$ is the rotation rate, $\nu$ is the kinematic viscosity of the solvent and $E$ is evaporation rate of solvent. By reverse engineering and using the measured thickness of the film, we can obtain the evaporation rate profile $E(\omega)$ for cases where a generalized model, based on Cregan's, holds. It is known that, for colloidal systems, the evaporation rate during spin-coating depends on parameters from the experiment and the suspension and on the rotation rate ( [24] and references therein

To generalize further the model to colloids, we have to take into account that the centrifugal force is essential to the spin-coating process, and matter (be it the solvent, or the colloidal particles) is what is expelled from the axis of rotation. Empty interstices 


\section{Pattern formation in magnetic spin-coating of hybrid colloids}

between colloidal particles of the deposit don't feel any force. Consequently, the left hand side of equation 1 should be substituted by the Compact Equivalent Height (CEH; see Fig. 4 in [23] and the corresponding text). It has been proven that under ample circumstances, this modified model holds for colloidal systems. Compact equivalent height can be obtained from the colloidal dried structure and its number of layers (or the occupation factor $\varepsilon^{2}$, for submonolayer systems), to which is proportionalfor the case of having only one kind of monodisperse colloidal particles.

Using the aforementioned proportionality, it is possible to obtain the ratio between the viscosity with applied field to the zero-field (standard) viscosity if we divide equation 1 for the two conditions. This leads to [23]:

$$
\frac{\nu(H, \omega)}{\nu(H=0, \omega)}=\left[\frac{\mathrm{CEH}(H, \omega)}{\operatorname{CEH}(H=0, \omega)}\right]^{3}=\left[\frac{\varepsilon^{2}(H, \omega)}{\varepsilon^{2}(H=0, \omega)}\right]^{3},
$$

where the right hand side usually is a measured quantity. This result holds even in the case where the particles are not monodisperse. Under some conditions, the model can be further generalized to cases where there are more than one kind of colloidal particles. The only change is that the expression that relates CEH with the measured occupation factor (resp. number of layers) and structure is more complex [24].

In this article, we report colloidal structures obtained in spin coating of hybrid colloids as a function of various experimental conditions, namely spinning speed, initial particle concentration, applied magnetic field strength and magnetic field configuration. We focus on the case of moderate initial concentration of ferromagnetic particles, where their magnetic interaction is relevant and the previously outlined models do not hold, i.e., an increase of effective viscosity is not the sole effect of the applied field.

\section{Experimental methods}

We perform the experiments in a commercial spin-coater (Laurell technologies, WS650SZ-6NPP/LITE/OND), wherein we built a pair of Helmholtz coils (Figure 1a) to apply magnetic fields in two main configurations depending on the electrical current carried by them. If both are equally-oriented, the substrate will spin in the region of uniform axial magnetic field (Figure 1b). Otherwise the substrate will be subjected to a radially oriented non-uniform field (Figure 1c).

In this work, we disperse carbonyl iron microparticles in cyclohexanone $\left(\mathrm{C}_{6} \mathrm{H}_{10} \mathrm{O}\right)$. Cyclohexanone has a density and evaporation rate similar to water (e.g. density: $\left.947 \mathrm{~kg} / \mathrm{m}^{3}\right)$. It is preferred to water because it presents a higher viscosity $(2.1 \mathrm{mPa} \cdot \mathrm{s}$, at røom temperature), which allows a slower sedimentation of the suspended particles. Carbonyl iron particles (Fe purity higher than $96.8 \%$, density $\rho=7.8 \mathrm{~g} / \mathrm{cm}^{3}$ ) were made by BASF SE (Germany) (grade EW). They are polydisperse showing a normallike shaped distribution with a degree of polydispersity of 0.7 , and a median diameter $\mathrm{d}_{50} \approx 3.0 \mu \mathrm{m}$. Carbonyl iron particles were coated by the manufacturer with a very thin layer of silica, to ease the re-dispersibility of the particles in suspension (see [26], and references therein). The resulting dispersion still is very unstable under sedimentation 
(see, e.g., [27] among others). To reduce even more the/ sedimentation rate of the magnetic particles in the suspension during the experiment, we also disperse much smaller (non-magnetic) silica particles $\left(\mathrm{SiO}_{2}, \rho=1.8 \mathrm{~g} / \mathrm{cm}^{3}\right.$, diameter $\approx 0.75 \mu \mathrm{m}$, degree of polydispersity smaller than 0.1 ) into it, to slightly increase the viscosity of the suspension [28-30]. We choose to use silica particles, because they are non-magnetic and with smaller density than iron. Other authors have used smaller carbonyl iron particles instead [31].

Glass substrates of an approximate size of $38 \times 25 \times 1 \mathrm{~mm}^{3}$ are used for all experiments. Firstly, they are cleaned with a diluted Micro-90 solution in an ultrasonic bath for fifteen minutes. Secondly, they are soft-etched for forty minutes by a basic piranha solution, which consists of ultra-pure water/ammonia/hydrogen peroxide with the ratio of 5:3:1 in volume at $67^{\circ} \mathrm{C}$. Thirdly, the substrates are thoroughly rinsed with ultra-pure water. Finally, they are dried by a filtered air blow. The resulting clean substrates are stored for $24 \mathrm{~h}$ before each experiment run, to homogenize their wetting properties.

In the suspensions, we use the same mass fraction of carbonyl iron particles and silica particles. They are weighed and homogeneously suspended in cyclohexanone to obtain a total particle concentration of $4.5 \%(\mathrm{v} / \mathrm{v})$. The individual volume fractions are 0.009 (iron), 0.036 (silica), and 0.955 (cyclohexanone). More diluted suspension of $1.5 \%(\mathrm{v} / \mathrm{v})$ was also used to show the effect of concentration on the results. The resulting batches are ultrasonicated for many hours and actively shaken before the experiments to ensure a well-dispersed suspension. Moreover, before each experiment run, the suspension is again put for $15 \mathrm{~min}$ in the ultrasonic machine and vigorously shaken. We set the spin-coater at the required spinning rate $\omega$ and applied magnetic field $H$. Once the substrate rotates at $\omega, 0.1 \mathrm{~mL}$ of suspension is pipetted onto the glass substrate. When the suspension dries or the system overheats, we turn off the rotation and the field. Micrographies of the dried deposits were taken from above with 


\section{Experiments with diluted colloids}

Experiments of spin-coating of ferromagnetic colloids at low concentrations (e.g. at 1.5\% $(\mathrm{v} / \mathrm{v}))$ with uniform axial applied magnetic fields, mainly lead to submonolayer deposits at low magnetic fields (see Figure 2 a for $29.5 \mathrm{kA} / \mathrm{m}$, for a typical image at a rotation rate of $2000 \mathrm{rpm}$ ). This can be understood under a simple model [25] modified to take the (hybrid) particulate nature of the fluid into account as well as a rotation rate dependent effective evaporation rate (see section 1 and [24]). Also, it has been shown that at those conditions, the effect of the magnetic field is only to increase the viscosity of the colloid. At higher magnetic fields there are regions of the substrate where multilayers are obtained (see Figure $2 \mathrm{~b}$ for $48.7 \mathrm{kA} / \mathrm{m}$, at the same rotation rate), but the model still holds fairly well [24]. This shows that the magnetic interaction between carbonyl iron particles is not strong enough to lead to noticeable non-linear effects like the formation of suprastructures in the considered time-scale (otherwise, the model would not hold).

a)

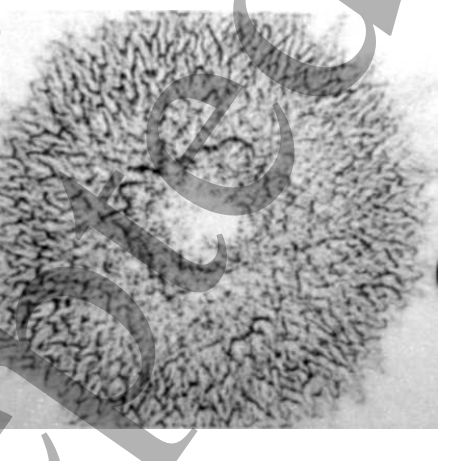

b)

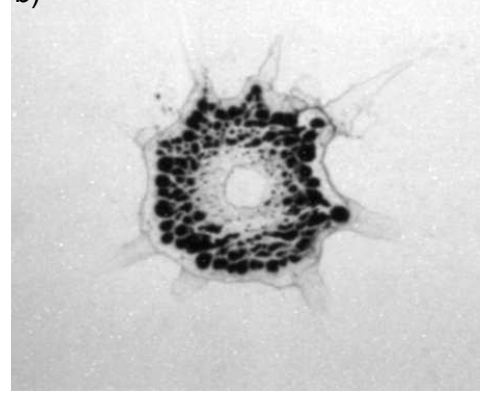

Figure 2. Photographs from above of dry deposits on the substrate from experiments of spin-coating of diluted $(1.5 \%(\mathrm{v} / \mathrm{v}))$ hybrid colloid under uniform magnetic field and a rotation rate of $2000 \mathrm{rpm}$. (a) Where magnetic field strength is $29.5 \mathrm{kA} / \mathrm{m}$ and (b) at higher magnetic field $(48.7 \mathrm{kA} / \mathrm{m})$. In both cases, the horizontal field of view is $12 \mathrm{~mm}$

Figure 3 shows the influence of increasing rotation rates on the behavior of spin coated deposits at an intermediate axial uniform magnetic field $(39.7 \mathrm{kA} / \mathrm{m})$. As rotation speed increases, there is a decreasing effect of surface tension effect, leading to an instability in the three phase moving contact line which can be observed in a less rounded front and more irregular patterns. 
Pattern formation in magnetic spin-coating of hybrid colloids
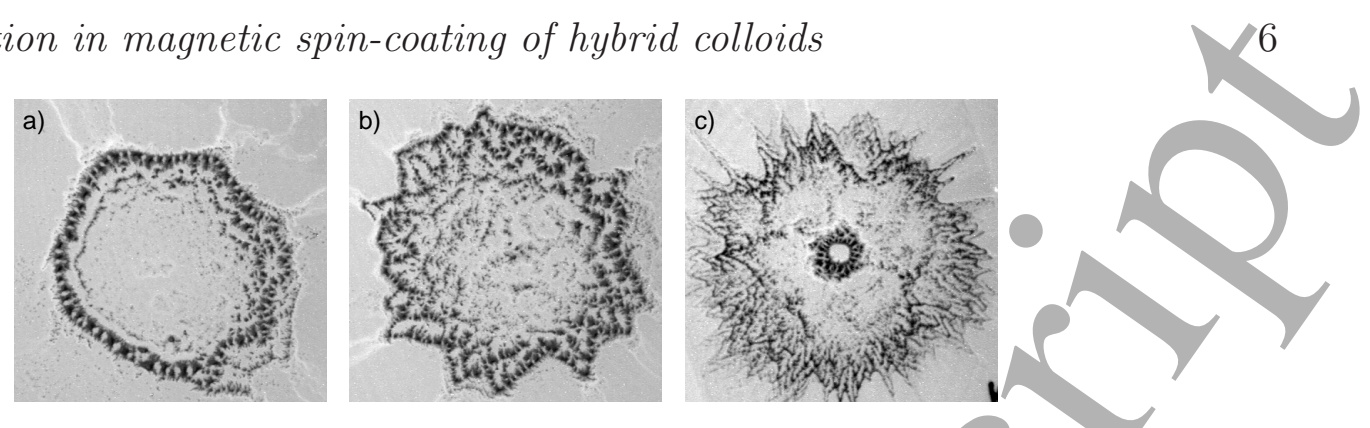

Figure 3. Photographs from above of spin-coated deposits from the experiments performed at uniform magnetic field with increasing spinning rates: a) $1300 \mathrm{rpm}, \mathrm{b}$ ) $1600 \mathrm{rpm}$ and c) $2400 \mathrm{rpm}$. In all cases, the concentration is $1.5 \%(\mathrm{v} / \mathrm{v})$, the magnetic field is $39.7 \mathrm{kA} / \mathrm{m}$, and the horizontal field of view $12 \mathrm{~mm}$.

A magnetic colloid with a higher particle concentration would overcome the weak dipolar interaction between the ferromagnetic particles and lead to clustering of the particles that allow the presence of more complex structures.

\section{Experiments with colloids at a higher concentration}

In this section, we show the main results of this work, which correspond to experiments performed at a $4.5 \%(\mathrm{v} / \mathrm{v})$ particle concentration, at different magnetic field configurations.

\subsection{Patterns at uniform axial magnetic applied fields}

In this section, we apply homogeneous axial magnetic fields during the spinning of the substrate (Figure 1b). At an intermediate magnetic field strength $(39.7 \mathrm{kA} / \mathrm{m})$, the interaction between the ferromagnetic particles is high enough to form suprastructures, which we call spikes (Figure 4). Spikes are magnetic dipoles formed by carbonyl iron particles, and are naturally oriented in the field direction in spite of the centrifugal force. This proves a high effective viscosity and an important magnetic interaction, which makes the previous models not applicable. Due to the centrifugal force, the spikes tend to appear at a given distance from the center of rotation. Moreover, we observe that the spikes form a quasi-one-dimensional array and are separated by a minimum distance which is due to an interplay between surface tension forces and the magnetic repulsion between equally oriented dipoles.

We measured the length of the spikes (and their distance to the axis of rotation) by using the translation stages of the microscope.

For small values of rotation speeds, we can observe a more regular disposition of the spikes around the center of rotation forming a circle (Figures 4a,b). At higher rotation rates, the uniformity of the circle breaks and leads to a less ordered structure of spikes as occurs similarly with the moving three-phase contact line in diluted colloids experiments (Figure 3). The small circle of spikes observed in figure 4c is due to a deviation of the suspension jet during pipetting in a form that it impinges onto the substrate slightly 

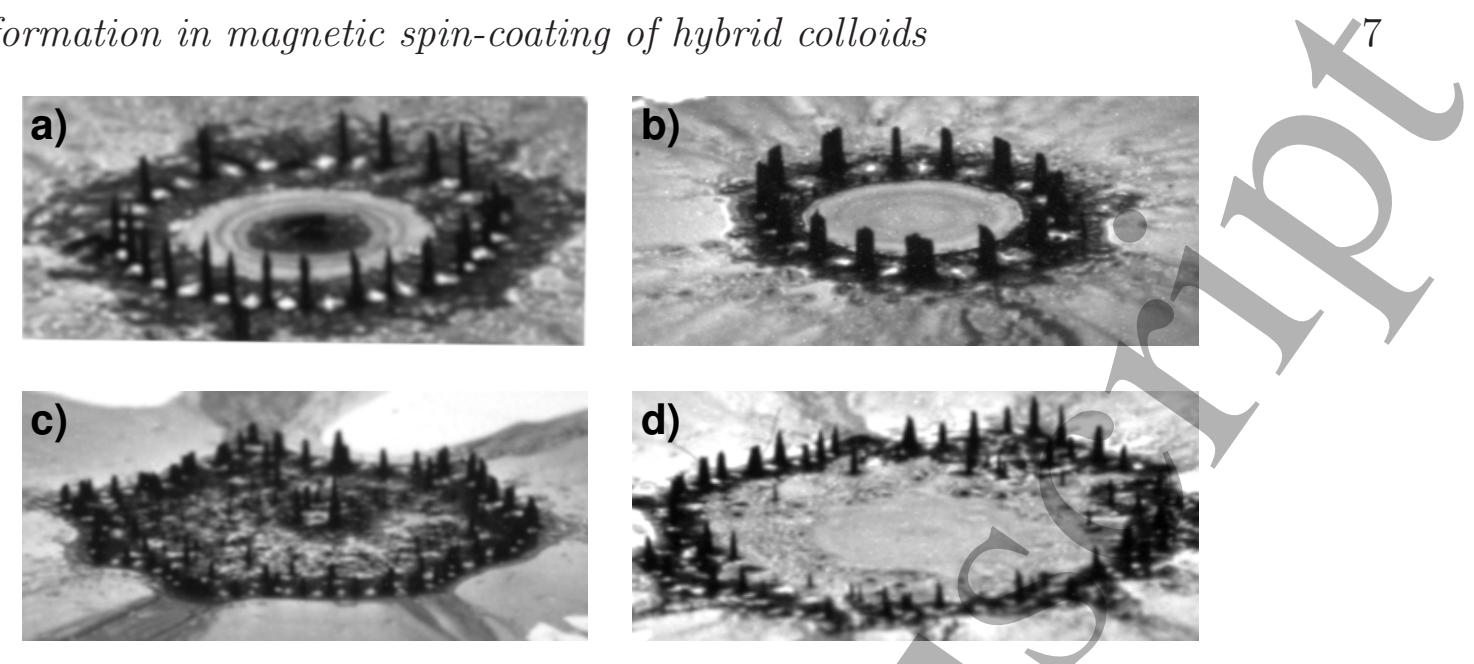

Pattern formation in magnetic spin-coating of hybrid colloids

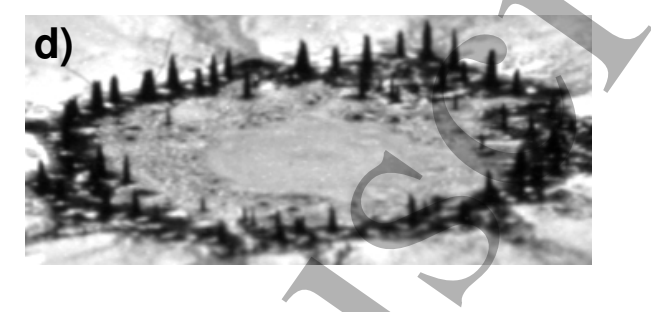

Figure 4. Photographs (lateral inclined view) of spin-coated deposits from the experiments performed at intermediate uniform magnetic field $(39.7 \mathrm{kA} / \mathrm{m})$ and a concentration of $4.5 \%(\mathrm{v} / \mathrm{v})$ with increasing spinning speeds $(\omega)$. a) $700 \mathrm{rpm}, \mathrm{b})$ $1200 \mathrm{rpm}$, c) $1600 \mathrm{rpm}$ and d) $2100 \mathrm{rpm}$. The horizontal field of view is $12 \mathrm{~mm}$.

off-center. We observed that these events, that happen time to time in our experiments, don't affect substantially the results from the big radii spikes, which are the source to calculate the data points in figure 5 .

We were able also to observe-spikes for rotation rates smaller than $700 \mathrm{rpm}$. However, at those frequencies the solvent didn't evaporate before the experiment finished, and consequently the spikes spread over the substrate once the field was switched off.

As rotation rate increases, the centrifugal force is higher and the average distance of the spikes to the center of rotation (radius) increases (Figure 5a). Also, the average length (height) of the spikes monotonously decreases (Figure 5b). At around $1200 \mathrm{rpm}$, the dispersion of spike length values is remarkably small and a local minimum of the distance of the spikes to the center of rotation can be observed. We think that this could correspond to a resonant frequency. Nevertheless, we neither understand the origin of this frequency nor the mechanisms involved in its selection. This will require further research and experiments.

\subsection{Patterns at non-uniform radial magnetic applied fields}

In this section, we apply a radial (non uniform) magnetic field during the spinning of the substrate (Figure 1c). Its maximum value is $3.5 \mathrm{kA} / \mathrm{m}$ and the average magnetic field gradient in the radial direction is $0.4 \mathrm{kA} / \mathrm{m}$ per millimeter. At low rotation speeds, it can be observed that the interaction between ferromagnetic particles leads to the formation of elongated dried deposits on the substrate. These deposits increase their average/length in the direction of the applied magnetic field as we move farther from the center of rotation (Figure 6).

In this magnetic field configuration, inertia (centrifugal force) shares its relevant direction with the applied field, allowing to an increase of the deposits length as the 


\section{Pattern formation in magnetic spin-coating of hybrid colloids}
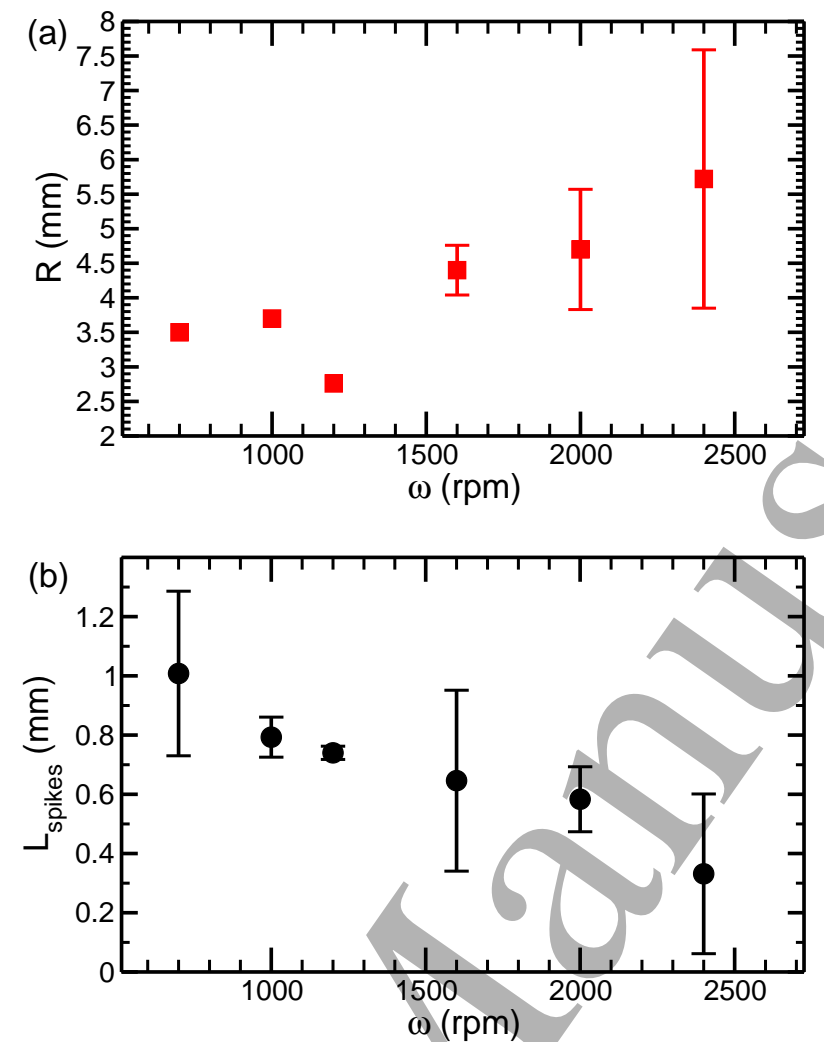

Figure 5. Spikes formation in spincoating of a $4.5 \%(\mathrm{v} / \mathrm{v})$ magnetic colloid, with an applied uniform magnetic field of $39.7 \mathrm{kA} / \mathrm{m}$, as a function of rotation rate $\omega$. (a) Average distance (radius) of the spikes to the center of rotation and (b) Length (height) of the spikes.

radius increases.

On the one hand, the typical magnetic field strength in this configuration is much lower $(1.8 \mathrm{kA} / \mathrm{m})$ than in the preyious one $(39.7 \mathrm{kA} / \mathrm{m})$, leading to a reduced magnetic effect. On the other hand, it has been previously shown that, for superparamagnetic particles colloids, the effective viscosity under the current configuration diminishes [32]. As a consequence, the typical length of the deposits are much smaller than the length of the spikes.

In figure 7 , the effect of the rotation rate on the length of the deposits for increasing radii is shown.

We don't observe a big change of the length of deposits for the narrow range of used rotation speeds. If we had used larger rotation speeds, there would have been less amount of colloidal particles available to cluster (equation 5 of [24]). Consequently, experiments at higher concentrations are needed to study the effect of the rotation speeds on the deposits for this magnetic field configuration. Higher concentrations and speeds would imply the need of using larger substrates. 


\section{Pattern formation in magnetic spin-coating of hybrid colloids}
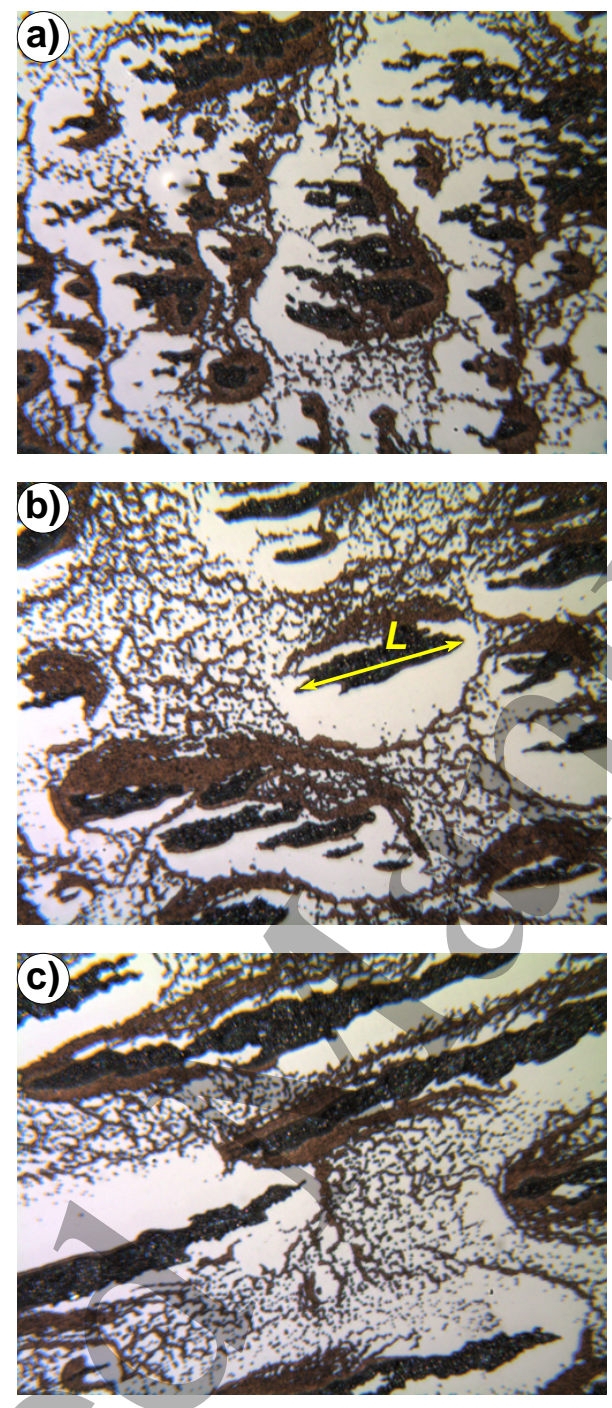

Figure 6. Micrographies (seen from above) of the spin-coated dried deposits of hybrid colloids at increasing distance from the center of rotation; a) $2 \mathrm{~mm}$, b) $6 \mathrm{~mm}$, and c) $12 \mathrm{~mm}$. The average (non-uniform) magnetic field is $1.8 \mathrm{kA} / \mathrm{m}$, the rotation rate is $700 \mathrm{rpm}$, and the HFOV is $0.66 \mathrm{~mm}$. The measurement of the length of the chains (dark deposits) is sketched in b) (double arrow).

\section{Concluding remarks}

In this article we apply magnetic fields during spin-coating of hybrid colloids where one constituent is ferromagnetic particles. We show that, at concentrations higher than a threshold, particles do not interact trivially and form chains / spikes which correspond to macroscopic magnetic dipoles.

If the applied magnetic field is uniform and in the direction of the rotation axis, we obtâin spikes which resemble the peaks appearing in Rosensweig instability for ferrofluids [33]. In their case but at our experiment conditions, the distance between peaks should be around $12 \mathrm{~mm}$. However, we observe an order of magnitude smaller. The discrepancy comes from the surface tension effect in Rosensweig instability which 


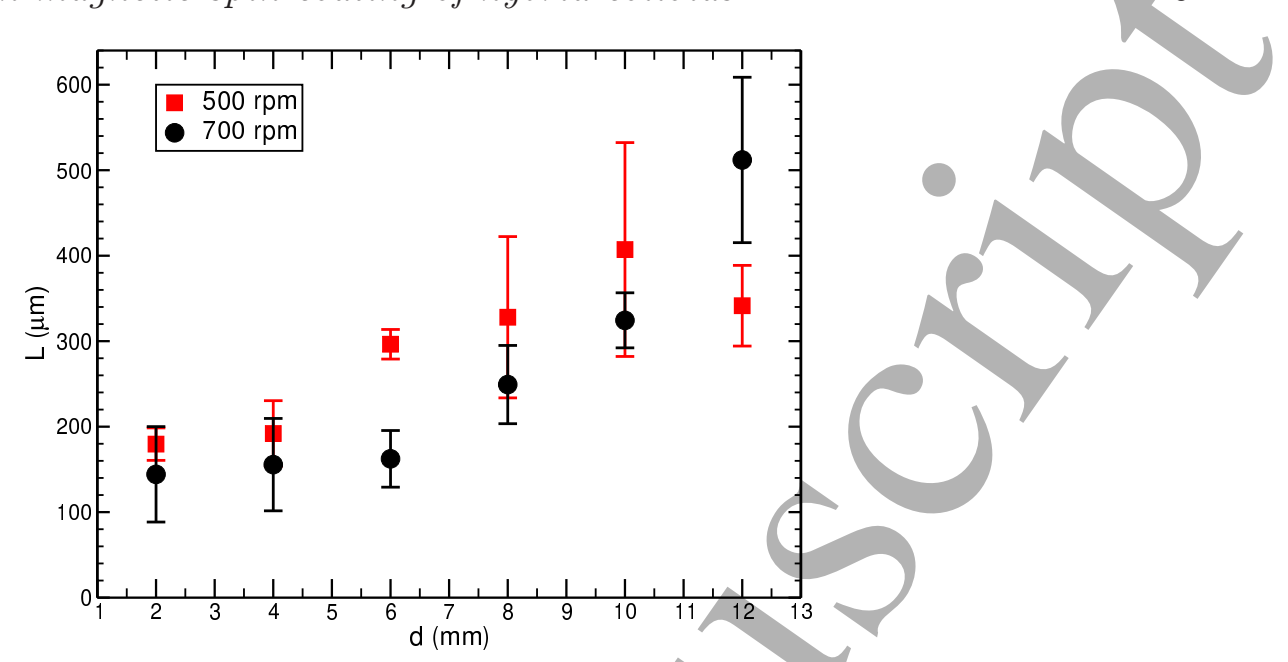

Figure 7. At nonuniform magnetic field the length of deposits chains (see figure 6) are shown as a function of the radius (distance to the center of rotation) for two different rotation speeds.

in fact determines the distance between the peaks. We think that, in our case, we are limited by the paramagnetic repulsion between the spikes and not by surface tension. In our experiment, surface tension plays an important role in the moving contact line front where at high rotation rates an instability develops that reduces the observed order in the disposition of the spikes around a circle. Moreover, the destabilizing mechanism in our set-up (rotation of the substrate) is not strong enough to orient the spikes in the direction of the centrifugal force, but to increase the distance of the spikes to the rotation center. On the other hand, the evaporation of the solvent freezes the spikes in the three phase contact line front when complete evaporation of the solvent occurs.

If the applied magnetic field is radial and not uniform, magnetophoresis induces a reduction of the effective viscosity as has been observed in previous works with superparamagnetic colloids [32]. The length of the structures (elongated deposits of particles) increases with the distance to the rotation center. The suspension, owing to its effective viscosity, is not confined in the substrate, and consequently we are not able to observe the effects of the aforementioned surface-tension induced instability.

We have shown that the effect on pattern formation of an axial uniform magnetic field is bigger than of a radial field. This is due to the fact that an enhanced viscosity gives the particles enough time to move and gather, to form clusters. Nevertheless, further research is required to identify the critical values of concentration which give rise to non-trivial structures like spikes, and the effect of the size distribution of the particles in the colloid. It is also important to characterize the transition between a purely rheological effect and others where different mechanisms may play a relevant role. This would be important to set limits to the application of the use of spin coating as a magnetorheometer, and also to tailor localized colloidal structures.

The quantitative effect of the magnetic interaction among the carbonyl iron particles when the simple model does not hold (i.e., at moderate or high volume 
1

2

3

4

5

6

7

8

9

Pattern formation in magnetic spin-coating of hybrid colloids

fractions) is still something to be understood. To achieve that, it will be required a comprehensive magnetic characterization of the particles and the suprastructures (spikes) themselves. We encourage further investigation in this direction.

\section{Acknowledgments}

This work was partially supported by the Spanish AEI (Grant nos. FTS2014-54101-P \& FIS2017-83401-P). We also thank Juan de Vicente for providing us with the carbonyl iron particles used in this work.

\section{References}

[1] Walker P H and Thompson J G 1922 P. Am. Soc. Test. Mater. 22465

[2] Emslie A G, Bonner F T and Peck L G 1958 J. Appl. Phys. 29 858-862

[3] Meyerhofer D 1978 J. Appl. Phys. 49 3993-3997

[4] Birnie III D and Manley M 1997 Phys. Fluids 9 870-875

[5] Acrivos A, Shah M J and Petersen E E 1960 J. Appl. Phys 31963

[6] Wahal S, Oztekin A, Bornside D, Brown R, Séidel P, Ackmann P and Geyling F 1993 Appl. Phys. Lett. 622584

[7] Furukawa H, Tsutsui H, Aoi K, Watanabe T and Nakamura I 2005 J. Phys.: Conference Series $14220-227$

[8] Bornside D E, Macosko C W and Scriven L E 1989 J. Appl. Phys. 66 5185-5193

[9] Hall D, Underhill P and Torkelson J 1998 Polym. Eng. Sci. 38 2039-2045

[10] Fraysse N and Homsy G M 1994 Phys. Fluids 61491

[11] Ohara T, Matsumoto Y and Ohashi H 1989 Phys. Fluids A 11949

[12] Sahu N, Parija B and Panigrahi S 2009 Indian J. Phys. 83 493-502

[13] Rehg T J and Higgins B G 1992 AIChE J. 38489

[14] Mihi A, Ocaña M and Míguez H 2006 Adv. Mater. 18 2244-2249

[15] Wu Y L 2007 Control over colloidal crystallization by shear and electric fields Ph.D. thesis Utrecht University

[16] Arcos Martínez C 2008 Preparación y Caracterización de Materiales Mesoestructurados Ph.D. thesis Facultad de Ciencias, Universidad de Navarra

[17] Giuliani M 2010 Colloidal crystal formation through interfacial mechanisms Ph.D. thesis University of Navarra URL http://hdl.handle.net/10171/13612

[18] Pichumani M, Bagheri P, Poduska K M, González-Viñas W and Yethiraj A 2013 Soft Matter 9 $3220-3229$

[19] Arcos C, Kumar K, Gonzáléz-Viñas W, Sirera R, Poduska K M and Yethiraj A 2008 Phys. Rev. E $77050402(\mathrm{R})$

[20] Aslam R, Ardanza-Trevijano S, Poduska K M, Yethiraj A and González-Viñas W 2017 Phys. Rev. E 950326007

[21] Pichumani M and González-Viñas W 2011 Magnetohydrodynamics 47191

[22] Bartlett A P, Pichumani M, Giuliani M, González-Viñas W and Yethiraj A 2012 Langmuir 28 3067

[23] Pichumani M and González-Viñas W 2013 Soft Matter 9 2506-2511

[24] Aslam R, Shahrivar K, de Vicente J and González-Viñas W 2016 Smart Mater. Struct. 25075036

[25] Cregan V and O'Brien S 2007 J. Colloid Interf. Sci. 314324

[26] Bombard A, Gonçalves F, Shahrivar K, Ortiz A and de Vicente J 2015 Tribol. Int. 81 309-320

[27] Cheng H, Zuo L, Song J, Zhang Q and Wereley N 2010 J. Appl. Phys. 10709 B507

[28] Einstein A 1906 Ann. Phys. 324 289-306 
Pattern formation in magnetic spin-coating of hybrid colloids

[29] Einstein A 1911 Ann. Phys. 339 591-592

[30] Batchelor G 1977 J Fluid Mech 83 97-117

[31] Ngatu G T and Wereley N M 2007 IEEE Trans. Magn. 43 2474-2476

[32] Aslam R and González-Viñas W 2017 Colloids Surf. A 532 530-534

[33] Andelman D and Rosensweig R E 2009 The phenomenology of modulated phases: from magnetic solids and fluids to organic films and polymers Polymers, liquids and colloids in electric fields: interfacial instabilities, orientation and phase transitions pp 1-56

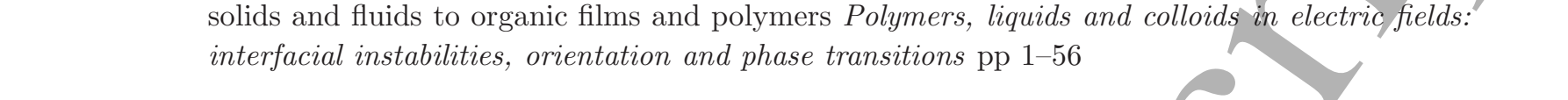

